\title{
娄Sociología
}

Maldifassi, J. (2018). Percepción social de la tecnología en Chile. Revista de Sociología 33(1), 39-57. doi: 10.535/0719-529X.2018.51107

\section{Percepción social de la tecnología en Chile}

\section{[Social perception of technology in Chile]}

\author{
José Maldifassi P. \\ Universidad Adolfo Ibáñez
}

\begin{abstract}
Resumen
El presente trabajo de investigación analiza cómo es percibida y conceptualizada la tecnología por la población adulta en Chile. Con este propósito, se diseñó un estudio cuantitativo con una muestra de 181 personas adultas habitantes de la Quinta Región de Valparaíso, Chile. Los resultados de la investigación muestran que el concepto de tecnología asume distintos significados conforme a la edad, sexo y grupo socioeconómico de las personas. Las mujeres tienden a asociar el concepto a la idea de internet y los hombres a procesos productivos complejos, en tanto que personas de estratos altos y medio-altos tienden a percibir la tecnología como algo más positivo que el resto de los grupos. A su vez, el Gobierno en tanto institución, es considerado por la mayoría de las personas como el agente principal del sistema nacional de innovación y el principal responsable de la generación de un desarrollo tecnológico genuino, seguido por las empresas y las universidades. Los resultados se analizan a fin de contribuir a la discusión respecto al desarrollo tecnológico en Chile
\end{abstract}

Palabras clave: tecnología, percepción de la tecnología, desarrollo tecnológico, aceptación social de la tecnología, género y tecnología.

\begin{abstract}
The present study analyzes how technology is perceived and conceptualized by Chile's adult population. A quantitative study was conducted based on a sample of 181 adult individuals resident in the region of Valparaíso, Chile. The results of the study show that the concept of technology is ascribed different meanings depending on the age, gender, and socioeconomic status of the individual. Women tend to associate the concept with the internet, while men think of complex manufacturing processes. Individuals of high and medium-high socioeconomic status tend to perceive technology more positively than other socioeconomic groups. Interestingly, in institutional terms, the Government is considered by the majority of individuals to be the leading agent within the national innovation system, seen as having the greatest responsibility in terms of generating genuine technological development, followed by private firms and universities. The analyzed results serve as a contribution to the discussion surrounding technological development in Chile.
\end{abstract}

Keywords: technology, perception of technology, technological development, social acceptance of technology, gender and technology.

Contacto: La comunicación sobre este artículo debe ser enviada a José Maldifassi, email: jose.maldifassi@uai.cl

Financiamiento: El autor agradece el financiamiento por parte del Programa de Apoyo a la investigación 2009 de la Facultad de Ingeniería y Ciencias de la Universidad Adolfo Ibáñez.

Agradecimientos: El autor desea agradecer a Victoria Valdebenito y al Dr. Roberto Arístegui del Departamento de Psicología de la Universidad Adolfo Ibáñez, por sus comentarios respecto a la versión preliminar de este artículo; y a Diego Santander por su apoyo en el procesamiento de datos. 


\section{INTRODUCCIÓN}

Según Bloor (1999) el conocimiento debe ser entendido en términos de la interacción entre una realidad independiente, el objeto del conocimiento, y un sujeto consciente, el cual posee sus propios principios de receptividad, quién, a partir de tales principios, construye representaciones del objeto en cuestión. Kotler, Armstrong, Cámara Ibáñez, y Cruz Roche (2004) sostienen que la percepción es aquel proceso mediante el cual las personas seleccionan, organizan e interpretan información para crear una imagen significativa del mundo. El llamado Teorema de Thomas (Thomas \& Thomas, 1928) establece que la definición que tienen las personas acerca de la realidad se transforma en el contexto y sus consecuencias, es decir, que la interpretación de la realidad por parte del individuo (lo que sería su percepción) es lo que condiciona el actuar del mismo en esa realidad subjetiva, transformándola así en realidad objetiva. Según esto, las personas actúan y reaccionan en base a sus percepciones acerca de la realidad y no necesariamente sobre la base de la realidad objetiva (Northcraft \& Neale, 1990). Coincidente con esto Berger y Luckmann (1997) sostienen que la realidad se construye socialmente a partir del cuerpo de conocimientos que posee la sociedad donde tal realidad es concebida, creándose así percepciones diferenciadas que afectan la comunicación entre individuos, creando espacios para el desentendimiento (Gordon, 1987). Como se sostiene desde la sociología del conocimiento (Merton, 1973) es evidente que tales percepciones estarán influenciadas por factores culturales, de género y derivados de la experiencia de vida de cada cual (Friedman,
2011; Gordon, 1987; Mannheim 1941). Por lo tanto, el comprender de mejor forma las percepciones que los individuos de una sociedad poseen acerca de ciertos hechos, problemas o conceptos (realidad) ayudará al proceso de comunicación y entendimiento entre los mismos, como también a comprender los posibles motivos que explicarían las condiciones existentes en la sociedad (contexto) relacionadas con tal realidad. Por tratarse la tecnología de un concepto abstracto, distintos individuos tienden a tener percepciones distintas acerca de tal concepto y también a definirlo de diferentes maneras (Flores, 1992; Rogers, 1995). Por ejemplo, Macionis (1989) define tecnología como "la aplicación de conocimiento cultural a la tarea de vivir en un ambiente físico" (p. 73), mientras que Basiuk (1977) la caracteriza como "una fuerza omnipresente, multifacética y dinámica, que tiene una elevada tendencia a provocar cambios en los entes sociales desde el más pequeño y local hasta el global" (p. 1). Por consistir entonces la tecnología en un elemento cultural (Macionis, 1989) y una forma fundamental del comportamiento humano (Pursell, 1990), esta afecta la vida diaria del individuo común. Como resultado de ello, cuando se lee o se escucha un mensaje que contiene el término tecnología, cada individuo tenderá a darle un significado diferente $y$, por ende, a interpretar el mensaje original de distinta forma. Con el propósito de ser capaces de transmitir claramente ideas y conceptos relacionados con la tecnología, y para poder comunicarse en forma efectiva con otros individuos en relación a este concepto, es necesario tener muy claro que es lo que cada individuo entiende por tecnología. 
Maldifassi, J. (2018). Percepción social de la tecnología en Chile. Revista de Sociología 33(1), 39-57. doi: 10.535/0719-529X.2018.51107

Consecuentemente, mediante el análisis de cómo la tecnología es percibida por distintos individuos, será posible comprender cuanto valor le otorgan ellas y ellos a productos tecnológicamente sofisticados, tales como computadores y teléfonos celulares, y a procesos sociales relacionados con la tecnología, tales como la innovación tecnológica, el cambio tecnológico, el desarrollo de tecnología autóctona y las políticas gubernamentales de incentivo al desarrollo de tecnología en general.

En toda sociedad el desarrollo de tecnología es responsabilidad compartida entre los individuos, las instituciones y el gobierno, en lo que se conoce como el Sistema Nacional de Innovación (Gill, Guasch, Maloney, \& Schady, 2005; Nelson 1993). De acuerdo a la cultura y a la estructura institucional particular de cada país, los individuos tenderán a asignar mayores, menores o distintos grados de responsabilidad a los agentes que operan al interior de tal sistema (Weinstein, 2002). La postura de cada país en términos de esta asignación social de responsabilidades tiene una enorme importancia para el desarrollo de tecnología autóctona, como también para las políticas públicas destinadas a incentivar la innovación. Dosi, Teece, y Chytry (1998) indican que las condiciones socio políticas en los países de origen de las grandes empresas tienen un impacto considerable en la velocidad y dirección de sus actividades tecnológicas. A su vez Gainza (2003) enfatiza la misma idea al sostener que "el desarrollo y difusión de las tecnologías dentro de una sociedad va a depender mucho de cómo la cultura valora la tecnología" (p. 132). Según Merton (1973) el desarrollo sustancial y persistente de la ciencia ocurre solo en las sociedades que proveen las condiciones tanto culturales como materiales para tal desarrollo, siendo posible extender esta misma afirmación al desarrollo de tecnología autóctona. Desde una perspectiva social una cultura asimila una tecnología cuando es capaz de integrarla a su universo de significaciones (Gainza, 2003). Esto puede ocurrir solo cuando los individuos poseedores de esa cultura incorporan el concepto tecnología y sus diversas acepciones y manifestaciones al conjunto de percepciones que constituyen ese universo de significaciones. Por su parte Pinch y Bijker (1987), enmarcados en la sociología constructivista, sostienen que la trayectoria de la tecnología y las características definitivas de los objetos tecnológicos son el resultado de un proceso de construcción social, que, mediante la negociación, el ejercicio del poder, las influencias culturales y las capacidades técnicas de los involucrados conducen a un cierre de tal proceso (Klein \& Kleinman, 2002). En una publicación posterior de Bijker (2009) y refiriéndose particularmente a la construcción social de la tecnología, este sostiene que la descripción de un artefacto por parte de diferentes grupos sociales relevantes para el fenómeno produce distintas descripciones $\mathrm{y}$, por ende, distintos artefactos. De este modo, las percepciones de los individuos en forma aislada, las actitudes de los mismos adoptadas a partir de tales percepciones, la cultura a nivel colectivo y los agentes nacionales involucrados en el desarrollo de tecnología influirán, positiva o negativamente, en el uso y adopción de diversas tecnologías, y en los procesos, productos y sistemas tecnológicos autóctonos que se desarrollen.

El modelo de aceptación de la tecnología (TAM por su sigla en inglés) desarrollado por Davis (1989) establece que las percepciones a priori que las personas poseen acerca de la utilidad de las 
tecnologías de información y comunicación afectan la aceptación y uso que hacen de tales tecnologías. De acuerdo con esto, las percepciones que posean los individuos acerca de la tecnología y el valor que asignen a las actividades tecnológicas y sus procesos relacionados, influenciarán la forma en que esos mismos individuos desarrollan, asimilan y usan las tecnologías, en como las empresas desarrollan y comercializan productos y procesos innovadores, y en cuan efectivas y eficientes son tales empresas en llevar a cabo actividades relacionadas con la innovación tecnológica. Asimismo, las políticas e incentivos del gobierno destinadas a impulsar el desarrollo tecnológico tendrán una distinta aceptación por parte de cada individuo, a partir de las percepciones que cada uno tenga acerca de la tecnología en general y los beneficios personales y sociales que de tales tecnologías se puedan derivar. Por consiguiente, el conocer las condiciones imperantes en Chile en cuanto a cómo los individuos definen, valoran y perciben la tecnología y sus procesos relacionados permitirá, en parte, explicar o comprender el porqué del atraso relativo de Chile en estas materias frente a países efectivamente más industrializados.

\section{Objetivos del estudio}

Si bien en Chile se han realizado estudios previos relativos a las percepciones sociales acerca de la tecnología, estos, en general, han tendido a centrarse en las tecnologías de información (Maldifassi \& Canessa, 2009; Silva-Peña, Borrero, Marchant, González, \& Novoa, 2006) y no han indagado en las percepciones de grupos sociodemográficos amplios, ni acerca del concepto específico tecnología. Teniendo esto en cuenta, el presente estudio tuvo como objetivo general el comprender de qué manera en Chile diferentes grupos sociodemográficos definen y perciben el fenómeno de la tecnología, cual es la importancia que le otorgan a la tecnología en sus vidas, y cómo perciben el rol de los distintos actores que operan dentro del Sistema Nacional de Innovación. El tema tiene especial relevancia ya que la comprensión de lo que es la tecnología y las actividades con ella relacionada es necesaria "para hacer juicios y elecciones en la vida diaria, mientras que una buena actitud de las personas hacia estas actividades permite trazar caminos que lleven a generar políticas tendientes a mejorar e impulsar dichas actividades" (Consejo Nacional de Ciencia y Tecnología de México [CONACYT], 1998, p. 1). Basado en los lineamientos de la sociología del conocimiento y de la teoría constructivista de la tecnología (Bijker 2009), el presente estudio pretende presentar el tema a partir de la realidad observada, a fin de comprender la influencia que tienen los factores sociodemográficos en la percepción de la tecnología y su aporte en la vida cotidiana. Estos resultados podrían aportar a una mejor comunicación sobre la tecnología y al desarrollo de estrategias de intervención que permitan mejorar la innovación tecnológica y su incorporación en la vida de los ciudadanos. 


\section{MÉTODO}

El presente estudio se circunscribe en una investigación cuantitativa, para la cual se diseñó una encuesta para evaluar las percepciones de la tecnología en Chile. La encuesta, aplicada de forma presencial, constaba de 10 preguntas, algunas de las cuales eran de elección múltiple y otras abiertas. Los encuestados se caracterizaron en base a su edad, sexo y grupo socioeconómico. En primera instancia se deseaba conocer los distintos significados que cada individuo otorga al concepto tecnología, ya que a partir de tal significado es que cada cual interpreta lo que en el entorno se menciona, identifica o asocia con tecnología.

Teniendo en cuenta que nuestra sociedad ha tendido a sobrevalorar todo lo relacionado con la tecnología, se indagó acerca de la importancia que cada individuo asigna a la tecnología y la utilidad que tiene la misma en la vida diaria de cada uno. En esta misma línea de análisis se le pidió a los entrevistados que expresasen su opinión acerca de la importancia que tendría la tecnología para los chilenos en general, esto último con el propósito de percibir la posible generalización de los beneficios que tendría la tecnología para la sociedad y permitir una comparación con los beneficios personales. Al ser Chile un país importador neto de productos que incorporan tecnología, interesaba conocer que opinión tendrían los encuestados respecto a esta realidad, ya que para algunos esto puede tener un efecto positivo y para otros, negativo. Relacionado con esto último y considerando los posibles efectos que el desarrollo tecnológico podría tener en la economía y el empleo nacional, se preguntó si el Gobierno debiera o no invertir más fondos públicos para desarrollar tecnología hecha en Chile.

Como el desarrollo tecnológico es un proceso que involucra diversos estamentos sociales, económicos y educacionales se preguntó a los entrevistados acerca de quién debiera ser responsable del desarrollo de tecnología, esto último con el propósito de avalar o no el hecho de que a nivel de gobierno o empresas se invierta más en desarrollar tecnología autóctona. Finalmente, se exploraron las ideas de los encuestados sobre las posibles causas del atraso tecnológico del país y de qué forma se podría mejorar la incorporación de tecnología en productos y procesos hechos en Chile.

\section{Muestra y Procesamiento de Datos}

La muestra fue obtenida a través de muestreo por conveniencia de población adulta urbana de Chile central, mediante la aplicación de encuestas en las ciudades de Viña del Mar y Valparaíso. El número total de encuestas válidas y analizadas fue de 181 casos que, asumiendo varianza máxima de proporciones y un $95 \%$ de confianza en la estimación del parámetro (Malhotra, 2004) permite un nivel de error asociado a la estimación de 7.3\%.

Cada uno de los casos de la muestra fue clasificado en tres variables sociodemográficas: sexo, edad y grupo socioeconómico de pertenencia. Con el propósito de evitar al 
entrevistado la necesidad de auto clasificarse dentro de un cierto nivel o grupo socioeconómico o de responder preguntas relativas a ingresos, la pertenencia a cada grupo fue determinada por el entrevistador, siendo esta clasificación la correspondiente a las clases baja, media-baja, media-alta y alta. Las características generales de la muestra se presentan en la Tabla 1.

Tabla 1. Características de la muestra

\begin{tabular}{|c|c|c|c|c|c|c|c|c|c|c|c|}
\hline \multirow{3}{*}{ Edad } & \multicolumn{8}{|c|}{ Grupo socioeconómico } & & & \multirow{3}{*}{ Totales } \\
\hline & \multicolumn{2}{|c|}{ Bajo } & \multicolumn{2}{|c|}{ Medio bajo } & \multicolumn{2}{|c|}{ Medio alto } & \multicolumn{2}{|c|}{ Alto } & \multicolumn{2}{|c|}{ Muestra } & \\
\hline & M & $\mathrm{H}$ & $\mathrm{M}$ & $\mathrm{H}$ & M & $\mathrm{H}$ & M & $\mathrm{H}$ & M & $\mathrm{H}$ & \\
\hline 20 a 30 & 5 & 5 & 4 & 5 & 9 & 8 & 5 & 9 & 23 & 27 & 50 \\
\hline 30 a 40 & 6 & 6 & 7 & 7 & 7 & 5 & 5 & 1 & 25 & 19 & 44 \\
\hline 40 a 55 & 14 & 3 & 4 & 8 & 7 & 9 & 6 & 4 & 31 & 24 & 55 \\
\hline Más de 55 & 5 & 3 & 4 & 4 & 4 & 3 & 4 & 5 & 17 & 15 & 32 \\
\hline \multirow{2}{*}{ Totales } & 30 & 17 & 19 & 24 & 27 & 25 & 20 & 19 & 96 & 85 & 181 \\
\hline & \multicolumn{2}{|c|}{$47(25.9 \%)$} & \multicolumn{2}{|c|}{$43(23.7 \%)$} & \multicolumn{2}{|c|}{$52(28.7 \%)$} & \multicolumn{2}{|c|}{$39(21.5 \%)$} & \multicolumn{2}{|c|}{181} & \\
\hline
\end{tabular}

Nota. Los valores tabulados son frecuencias. Porcentajes se muestran entre paréntesis. $\mathrm{M}=$ mujeres. $\mathrm{H}=$ hombres.

Conforme a los porcentajes indicados en la Tabla 1 y teniendo en cuenta las características sociodemográficas de la sociedad chilena (INE, 2013), es posible indicar que la muestra en términos demográficos es una adecuada representación de los chilenos en general, aun cuando es posible que algunos grupos estén sobre o subrepresentados. El rango de edad de los encuestados se eligió esperando una representatividad de adultos más que de jóvenes. Los datos obtenidos fueron analizados con el software SPSS 19 empleando estadística descriptiva bivariada y multivariada para agrupar respuestas en conjuntos homogéneos diferenciados, que permite crear una función predictiva de las variables dependientes a partir de las variables independientes.

\section{RESULTADOS}

Imágenes de la Tecnología

Al consultársele a los entrevistados por el significado de la palabra tecnología y dándoles la oportunidad de elegir todas las acepciones posibles de un conjunto de alternativas, en orden de mayor a menor preferencia las elecciones fueron las siguientes: computadores (57.5\%), Internet (55.2\%), conocimientos avanzados (49.7\%), maquinaria (47.5\%), procesos productivos complejos (38.7\%), y nuevos productos más complejos (34.3\%). Se percibe en este orden de preferencias una evidente influencia de lo que son las nuevas tecnologías 
informáticas, las cuales se encuentran muy cerca de la vida diaria de todas las personas. Tales preferencias responden también a la conceptualización que actualmente la publicidad de los equipos informáticos tiende a asociar con la palabra tecnología.

Se analizó la relación de estas definiciones de tecnología entre sí, comprobándose que hay tres conceptos genéricos claramente diferenciados que las agrupan, estos son: informática y maquinaria, procesos y productos complejos, y conocimientos avanzados. El uso de solo uno de estos conceptos genéricos tendería a excluir los otros dos, no debiéndose producir ambigüedades ni mal entendidos en la percepción por parte de la sociedad del concepto tecnología aludido. Ante la oportunidad otorgada a los entrevistados para que dieran espontáneamente otras definiciones de tecnología se obtuvo las respuestas que se muestran en la Tabla 2.

\section{Tabla 2. Definiciones de tecnología según los propios encuestados}

Procesos productivos más fáciles.

Automatización y abaratamiento de costos productivos.

Perfección del entorno.

Desarrollar cosas difíciles con un grado de rapidez y eficacia.

Procesos o instrumentos que mejoran la producción.

Realización de trabajos en menor tiempo.

Biotecnología, alimentos transgénicos.

Palabra que nos hace más fácil la vida.
Crecimiento económico, productividad, eficiencia.

Productos con un nivel tecnológico superior al normal.

Conocimientos técnicos avanzados.

Estudios de cómo llevar a la práctica el conocimiento científico.

Objetos creados a través de la intervención del ser humano.

Todos los mecanismos, sistemas y procesos que facilitan el desenvolvernos mejor.

Celulares / Teléfono

China.
De la Tabla 2 cabe destacar la variedad y riqueza de las definiciones planteadas por los encuestados para un concepto abstracto que se emplea habitualmente. $\mathrm{Si}$ se hace un agrupamiento conceptual de las diversas definiciones espontáneas de tecnología que se muestran, se concluye que abarcan nociones similares a los conceptos genéricos identificados arriba, tales como: procesos industriales avanzados, productos avanzados, conocimientos avanzados, bienestar y facilidad de vida, y desarrollo económico. De acuerdo con esto no existiría genéricamente la tecnología, sino que a nivel de la sociedad existen cognitivamente muchas tecnologías en forma simultánea, ya que son diversas las definiciones y percepciones acerca de la misma que poseen los individuos, dándose lugar a lo que Law (2002) define como "la coherencia fraccional simultánea de múltiples objetos y sujetos" (p. 4) para un mismo 
fenómeno, ninguno de los cuales se puede considerar como correcto o incorrecto. Lo anterior indicaría que en futuros estudios relacionados con este tema se deberá incorporar un conjunto más amplio de definiciones de tecnología que el empleado en la presente investigación.

$\mathrm{Al}$ analizar los resultados de acuerdo con sexo de los encuestados, se encontró que los hombres, en una mayor proporción que las mujeres, se inclinaron por definir tecnología como procesos productivos complejos. Las mujeres eligieron como definición de tecnología Internet en una proporción superior que los hombres. Esta última preferencia (Internet) también resultó ser mayor entre los encuestados de nivel socioeconómico bajo en comparación con los otros tres niveles. Además de esto, el grupo socioeconómico alto presentó un nivel de preferencias superior que los otros grupos por procesos productivos complejos. Lo anterior indicaría que las mujeres de estrato socioeconómico bajo tienden a definir tecnología como Internet, mientras que los hombres de estrato socioeconómico alto tienden a definir tecnología como procesos productivos complejos. Esto sugiere que, al emplear el concepto tecnología en cualquier contexto, es necesario indicar previa y explícitamente la definición concreta de tecnología a la cual se hace referencia, para asegurar un entendimiento adecuado del mensaje que se desea comunicar.
Importancia de la Tecnología

Respecto a la importancia que tiene la tecnología en sus vidas, los resultados muestran una clara tendencia a considerar la tecnología como un factor importante en la vida de las personas, tal como se aprecia al presentar de mayor a menor preferencia los porcentajes de las alternativas elegidas: alta (34.3\%), muy alta (31.5\%), media (23.8\%), baja (9.4\%). Cabe destacar que un 65.8\% de los entrevistados percibe la tecnología como de alta o muy alta importancia para su vida, lo cual se considera congruente con las condiciones de vida de la sociedad urbana actual. Llama la atención que un $9,4 \%$ de los entrevistados considera que la tecnología tiene una baja importancia en su vida, lo que debiera ser analizado con mayor detalle en futuras investigaciones.

A su vez, se encontró una diferencia estadísticamente significativa entre hombres y mujeres, dándole los primeros una importancia entre alta y muy alta (79.7\%) a la tecnología en sus vidas, mientras que las mujeres le dan una importancia entre media y alta (68.4\%). En términos de clase socioeconómica, el grupo alto tiende a otorgar mayor importancia a la tecnología en sus vidas que los grupos más bajos. De acuerdo con esto, las mujeres de clase socioeconómica baja tienden a otorgar menor importancia a la tecnología en sus vidas que los hombres de clase socioeconómica alta.

Ante la pregunta acerca de qué es lo que la tecnología permite en la vida de cada uno, el orden de preferencias otorgado por los encuestados en este caso fue el siguiente: hacer 

tecnología en Chile. Revista de Sociología 33(1), 39-57. doi: 10.535/0719-529X.2018.51107

las cosas en forma más fácil (79\%), hacer las cosas en forma más simple (57.5\%), hacer más cosas $(56.4 \%)$, estar comunicado más fácilmente (56.4\%), hacer las cosas en forma más barata (30.4\%), estar mejor de salud (26\%), hace la vida más cara $(11.6 \%)$, y hace la vida más difícil (7.7\%). Cabe destacar la percepción positiva que en general tienen los entrevistados respecto a lo que la tecnología les permite en sus vidas, sin dejar de mencionar que un grupo importante de ellos tiene una visión negativa respecto de la misma (17.7\%), coincidente con el hallazgo de baja importancia de la tecnología en la vida para un cierto grupo de individuos mencionado arriba. Los individuos de los grupos socioeconómicos alto y medio-alto consideran que la tecnología les permite hacer más cosas (65.9\%) en mayor proporción que las personas de los grupos socioeconómicos bajo y medio-bajo (46.7\%). En el caso de aquellos que sostienen que la tecnología les permite hacer las cosas en forma más fácil, dicha opción fue preferida por los grupos socioeconómicos alto y medio-alto (86.8\%) por sobre los grupos bajo y medio-bajo (71.1\%). Asimismo, las personas de los grupos socioeconómicos alto y medio-alto tienden a consideran que la tecnología les permite hacer las cosas en forma más barata en una mayor proporción (43.9\%) que los de grupos socioeconómicos bajo y medio-bajo (16.7\%).

Por su parte, los hombres (37.6\%) tienden a considerar por sobre las mujeres $(23.9 \%)$ que la tecnología les permite hacer las cosas en forma más barata. Fue también posible comprobar que los encuestados de los grupos socioeconómicos alto y medio-alto consideran que la tecnología les permite hacer las cosas en forma más simple, en mayor proporción $(69.2 \%)$ que los entrevistados de los grupos bajo y medio-bajo (45.5\%). Respecto a la afirmación que señala que la tecnología permitiría estar mejor de salud, los grupos alto y medio-alto demostraron una mayor preferencia (62.6\%) por esta proposición que los grupos bajo y medio-bajo (50\%).

Por lo tanto, los individuos de los grupos socioeconómicos alto y medio-alto tienden a percibir en una mayor proporción que las personas de los grupos bajo y medio-bajo, que la tecnología les permite: hacer más cosas, hacer las cosas en forma más fácil, más simple y más barata, junto con estar mejor de salud.

Estos hallazgos indicarían que la tecnología es un fenómeno cuya percepción de ser positiva en la vida diaria estaría asociada principalmente con los grupos socioeconómicos alto y medio alto, en desmedro de los grupos bajo y medio-bajo. La explicación para esto puede ser dada en términos económicos, educacionales y laborales. El acceso a tecnología de mayores prestaciones y de mejor calidad es relativamente caro, por lo cual su adquisición y beneficios derivados se ven limitados para los individuos de las clases sociales baja y media-baja. Para poder emplear la tecnología más sofisticada se requieren niveles crecientes de educación y conocimientos, a los cuales las personas de las clases sociales baja y media-baja tienen menor acceso. En los lugares de trabajo la incorporación de tecnología más sofisticada históricamente ha desplazado a los trabajadores menos calificados y ha hecho el trabajo más demandante en términos intelectuales y de conocimiento. Esto llevaría a 
que los grupos socioeconómicos de menores ingresos perciban la tecnología en términos mucho menos favorables que los individuos de los grupos socioeconómicos alto y medio-alto.

$\mathrm{Al}$ realizar un análisis de regresión lineal múltiple, fue posible determinar que la variable que más influye en asignar importancia a la tecnología en la vida de cada cual es grupo socioeconómico, seguido de sexo y finalmente la edad. Esto se puede comprobar según se muestra en la Tabla 3.

Tabla 3. Parámetros de la regresión lineal múltiple

\begin{tabular}{ccccc}
\hline Parámetro & $\mathrm{B}$ & $\mathrm{SE}$ & t de Student & Valor-p \\
\hline Intercepto & 3.712 & 0.207 & 17.928 & $<.001$ \\
Hombre & 0.524 & 0.137 & 3.821 & $<.001$ \\
Edad 20-29 & 0.333 & 0.192 & 1.738 & .084 \\
Edad 40-55 & 0.034 & 0.185 & 0.184 & .854 \\
Mas de 55 & 0.142 & 0.213 & 0.666 & .506 \\
Grupo L & -0.753 & 0.199 & -3.787 & $<.001$ \\
Grupo P & -0.133 & 0.203 & -0.655 & .513 \\
Grupo Q & -0.013 & 0.193 & -0.065 & .948 \\
\hline
\end{tabular}

Nota: Variable dependiente = Importancia de la tecnología en la vida. $\mathrm{B}=$ parámetro estimado (no estandarizado) de regresión lineal. $\mathrm{SE}=$ error típico. Grupo $\mathrm{L}=$ nivel socioeconómico bajo, Grupo $\mathrm{P}=$ nivel socioeconómico medio, Grupo $\mathrm{Q}=$ nivel socioeconómico medio-alto. Categoría de referencia $=$ Grupo $\mathrm{R}$ (nivel socioeconómico alto). $\mathrm{B}=$ coeficiente de regresión estimado. $\mathrm{SE}=$ error típico.

Como condición basal del modelo de la Tabla 3 se creó un modelo con las variables mujer, edad 30-39, nivel socioeconómico alto (Grupo R). Esta condición base corresponde al valor calculado como Intercepto en el modelo. Es decir, un caso base presenta una importancia de la tecnología en la vida de 3.712 sobre un máximo de 5 (muy importante). Este intercepto es estadísticamente significativo a un nivel de confianza de 95\%. Si en lugar de que el caso particular a analizar sea de género femenino, al puntaje del intercepto se le debe agregar un valor 0.524, resultando en una importancia para tal caso de Hombre, Edad 30-39, grupo R un valor de 4.236. Este valor también es estadísticamente significativo a nivel $\alpha<.05$, lo que implica que, estadísticamente, los hombres le asignan a la tecnología mayor importancia en la vida que las mujeres. En relación con la edad, los coeficientes de la regresión $\mathrm{Bi}$ para todos los rangos de edad diferentes del caso base Edad 30-39 no son estadísticamente significativos con $\alpha<.05$. La única excepción sería Edad 20-29 que llegaría a ser estadísticamente significativo si el nivel de aceptación se relajara a $\alpha=.1$. Hacer esto no significaría ser mucho más exigente $y$, estadísticamente, significaría que las personas más jóvenes de la muestra le otorgarían 0.333 puntos adicionales a la importancia de la tecnología en la vida que el caso base (30-39 años).

En relación con nivel socioeconómico la explicación es la siguiente. Si en lugar de que 
el individuo a analizar perteneciera al grupo $\mathrm{R}$ (nivel socioeconómico alto) del caso base, lo hiciera perteneciendo al nivel medio-alto Grupo $\mathrm{Q}$, al nivel base de importancia de la tecnología del intercepto 3.712 habría que restarle 0.013 puntos. Este valor del parámetro es no significativo, lo mismo que para el grupo $\mathrm{P}$, lo que implica que los grupos medio-alto (Q) y medio (P) no tienen una diferencia estadísticamente significativa en la importancia que le otorgan a la tecnología en comparación con el grupo de referencia alto (R). A diferencia de lo anterior, si el individuo a analizar perteneciera al grupo socioeconómico bajo (L), al valor base del intercepto 3.712 habría que restarle 0.753 puntos, resultando que el caso mujer, edad 30-39, Grupo L, le otorgaría a la importancia de la tecnología en la vida un valor solamente de 2.959. Lo importante de esto es que este efecto de pertenecer al Grupo L y otorgarle una menor importancia a la tecnología en la vida que los otros grupos socioeconómicos es estadísticamente significativo. Este último hallazgo es muy relevante ya que corrobora la concepción de Karl Marx de que la tecnología es parte de la superestructura capitalista (Roth 2010) y que coopera a la alienación de los individuos (Wendling 2009).

Ante la pregunta acerca de la importancia que se le atribuye a la tecnología en la vida de cada uno de los entrevistados, las respuestas fueron las siguientes: alta o muy alta (65.8\%), media $(23.8 \%)$, baja $(9.4 \%)$. Con un porcentaje levemente inferior a la pregunta de beneficio personal de la tecnología y congruentemente con ella, un $64.7 \%$ de los entrevistados considera que la tecnología tiene una importancia alta o muy alta para los chilenos en general. Cabe destacar que ante esta pregunta el porcentaje de individuos que indica una baja importancia general de la tecnología (6.1\%) es menor que para la pregunta de importancia personal, lo que podría indicar que para aquellos que consideran que la tecnología tiene una baja importancia en sus vidas, esta tendría un efecto de mayor beneficio social más que personal, esto es, no tan bueno para mí pero mejor para el país en general. Esta percepción de mayor beneficio de la tecnología para las personas en general por sobre el beneficio personal fue también encontrada en Chile específicamente para las tecnologías de información (Maldifassi \& Canessa 2009).

\section{El Sub-desarrollo Tecnológico de Chile}

En relación a la opinión de los sujetos sobre la afirmación de que la tecnología que se emplea en Chile es casi toda importada, las respuestas tendieron a concentrarse en las categorías negativas: es malo porque no se crea tecnología chilena $(68 \%)$, es malo porque no se crean empleos (49.7\%), es bueno porque es más barato $(23.8 \%)$, es malo porque no se puede exportar $(23.8 \%)$, es malo porque es más caro (21\%).

La alternativa de mayor preferencia, es malo porque no se crea tecnología chilena, sugiere la idea de que a los entrevistados les gustaría que existieran los medios para generar más tecnología localmente. Al mismo tiempo puede 
ser interpretado como un indicador de un cierto nacionalismo o chovinismo, el que en ocasiones puede asomar (como en este tipo de encuestas), ya que para quedar bien con el entrevistador el encuestado denota su preferencia por lo chileno, lo cual no necesariamente puede ser cierto. La segunda alternativa más preferida, es malo porque no se crean empleos, también muestra la preocupación y conciencia de que si se contara con mayor tecnología propia, Chile no tendría tanta necesidad de importar productos, como también que podría exportar productos y servicios más innovadores, lo cual podría generar más empleos. Las personas mayores de 40 años tienden a considerar en mayor proporción (33.3\%) que los más jóvenes (14.9\%) que el hecho de que casi toda la tecnología que se emplea en Chile es importada es malo ya que no permite exportar. Por esto, las personas de mayor edad verían de mejor forma que los jóvenes el desarrollo de tecnología nacional en apoyo a las exportaciones.

Para analizar el rol que le compete al Estado en el desarrollo de tecnología se consultó a los encuestados si sería correcto que el gobierno destinara más fondos al desarrollo de tecnología hecha en Chile. Ante esta pregunta las respuestas de los encuestados fueron categóricas ya que el $87.8 \%$ de los encuestados señaló que sí se debieran destinar más fondos fiscales al desarrollo de tecnología nacional, contra un $7.7 \%$ que dijo que no se deberían destinar más fondos, y un $4.4 \%$ que no supo o no quiso contestar la pregunta. Entre las razones que algunos de los entrevistados dieron acerca de porque el gobierno sí debiera apoyar el desarrollo de tecnología nacional (pregunta de respuesta abierta), destacan las que se incluyen en la Tabla 4.

Tabla 4. Razones por las cuales el gobierno debiera invertir más en tecnología hecha en Chile

El país está dependiendo mucho de tecnología externa, que llega a altos precios y se debiera disponer de tecnología en forma más accesible para los chilenos.

No se está preparando a personas hoy en día que asuman un rol protagónico para el desarrollo tecnológico y esto debiera apoyarlo el gobierno.

Hay mucho potencial que se pierde por falta de financiamiento para la investigación.

Es importante para el desarrollo de un país.

Es importante para la educación.

Para mejorar la salud.
Es la forma de vender bienes y servicios con mayor valor agregado, generándose ventajas competitivas y abriendo nuevas ventanas a la exportación.

Así se le podría dar más facilidades a las Universidades para que trabajen en investigación.

El gobierno tiene los recursos como para impulsar el desarrollo tecnológico.

Es importante para el crecimiento económico.

Se generaría más empleo. 

tecnología en Chile. Revista de Sociología 33(1), 39-57. doi: 10.535/0719-529X.2018.51107

La opinión de mejorar el desarrollo del país a través de la tecnología fue la razón más frecuente, con especial énfasis en las oportunidades laborales y en la educación, como también para competir con otros países. Esto refleja una opinión en general positiva al desarrollo de tecnología nacional por parte del gobierno para apoyar los esfuerzos en pos del desarrollo social y económico del país. Quienes sostienen que el gobierno no debiera apoyar este tipo de iniciativas con más financiamiento, señalan como razones las siguientes: (a) Hay cosas más prioritarias en qué invertir hoy en día en Chile; (b) No es el gobierno sino las empresas las que debieran invertir más; (c) No depende solo del gobierno; (d) La tecnología no es necesaria; y (e) En Chile no existe la capacidad intelectual. $\mathrm{Si}$ bien el porcentaje de entrevistados que contestó que no a esta pregunta es muy bajo (solamente un 7.7\%), llama la atención las dos últimas respuestas, ya que de ellas se desprende una percepción altamente negativa acerca de la necesidad de la tecnología y la capacidad de crearla con medios nacionales.

Los Responsables del Desarrollo Tecnológico

En relación a la responsabilidad que le cabría a los distintos agentes nacionales el desarrollo de tecnología autóctona, las preferencias se inclinaron fuertemente hacia tres alternativas que fueron marcadas por más del $60 \%$ de los encuestados. De estas el gobierno acaparó la mayoría de las preferencias (77.9\%), seguido por las empresas (64.1\%) y las universidades $(61.3 \%)$.
No se encontraron diferencias estadísticamente significativas entre grupos al momento de considerar si el gobierno debiera ser el principal responsable del desarrollo de tecnología ya que todos los grupos apoyaron esta alternativa. Algo similar ocurre en el caso de las universidades, donde el apoyo a esta alternativa de desarrollo de tecnología es relativamente generalizado. Los laboratorios de investigación nacionales obtuvieron un $49.7 \%$ de las preferencias, lo cual también la hace ser una alternativa relevante, a pesar de que el número de estos establecimientos a nivel nacional es bastante reducido (Weinstein, 2002). Los inventores independientes obtuvieron un nivel de preferencias bajo (24.9\%), consecuente con el hecho de que a nivel nacional estos son casi una curiosidad por su bajo número y escaso aporte real a la innovación local.

En términos de los grupos socioeconómicos, los grupos alto y medio-alto tienden a preferir que sean las empresas y los laboratorios nacionales los responsables del desarrollo de tecnología en Chile. Al considerar la alternativa de que sería responsabilidad de los inventores independientes el desarrollo de tecnología en Chile, esta alternativa fue marcada en mayor proporción por los encuestados del grupo socioeconómico alto $(41 \%)$, resultando superior a la preferencia de los otros grupos (promedio de los otros tres grupos 20.3\%). Cabe destacar que para el grupo socioeconómico alto cada una de las alternativas propuestas fue considerada como importante, lo que indicaría para este grupo una percepción de que todos los agentes del Sistema Nacional de Innovación tienen un rol 
significativo que cumplir en la labor de desarrollar tecnología nacional.

La información anterior indicaría un apoyo sustantivo a la labor del gobierno en el desarrollo de tecnología en Chile, con un menor énfasis en que también sean las empresas y las universidades quienes desarrollen tecnología local. Menor consenso se obtiene en el caso de los laboratorios nacionales e inventores independientes. Por lo anterior, los datos indican que la labor de generar tecnología a nivel nacional debiera ser una preocupación conjunta de los diversos agentes del Sistema Nacional de Innovación, con especial énfasis en el rol prioritario del gobierno. Este hallazgo tendería a favorecer los programas de incentivo a la innovación tecnológica que se han impulsado por los últimos gobiernos, que han otorgado más fondos del Estado para investigación y desarrollo en empresas y universidades, por ejemplo, centrados en CONICYT y CORFO. Esto contrastaría con la realidad de los países desarrollados, en los cuales el gobierno tiene un rol secundario en comparación a la actividad principal de las empresas en lo que a investigación, desarrollo e innovación tecnológica respecta (Organization for Economic Cooperation and Development [OECD], 2013).

Con respecto a qué le faltaría a Chile para aumentar el nivel tecnológico de los procesos de manufactura y productos hechos en el país, los encuestados respondieron que lo que haría falta es un mayor aporte en dinero por parte de las empresas $(65.7 \%)$ y más dinero aportado por el gobierno (64.1\%). Nuevamente hubo consenso respecto a que el gobierno debiera invertir más dinero para estos fines, ya que no fue posible discriminar entre los grupos analizados, debido a que la gran mayoría de los encuestados respondió que sí a esta alternativa.

Los encuestados del grupo socioeconómico alto consideran que las empresas debieran invertir más dinero en el desarrollo de tecnología de productos y procesos, en una proporción mayor que los otros grupos. A diferencia de la pregunta anterior donde la mayor cantidad de preferencias la obtuvo el gobierno, llama la atención el hecho que en esta pregunta el mayor número de preferencias se lo adjudicaron las empresas, siendo que ambas preguntas apuntan al mismo tema de la responsabilidad al momento de desarrollar tecnología nacional. En parte la diferencia entre la pregunta anterior con esta podría deberse a que esta última pregunta se refiere explícitamente a procesos de manufactura y productos, los cuales obviamente se relacionan en forma específica con el quehacer de las empresas más que con el gobierno. En tercer lugar, dentro de las preferencias para desarrollar tecnología chilena se ubicó la existencia de una mayor cantidad de laboratorios nacionales (48.1\%).

Las opiniones de los encuestados indicarían que la falta de personal calificado para aumentar el nivel tecnológico nacional sería menos relevante que las causas ya mencionadas, considerando que un $42.5 \%$ de ellos indicó que lo que faltaría serían más técnicos, un $37 \%$ que faltarían más científicos, un $30.4 \%$ que faltarían más ingenieros y un $21 \%$ que faltarían más inventores independientes. Asimismo, los grupos socioeconómicos alto y medio-alto tienden a 
sostener que lo que falta son más científicos (43.9\%), mientras que los mayores de 40 años sostienen que lo que faltan son técnicos (50.6\%). Respecto a que faltarían más ingenieros, no hubo diferencias estadísticamente significativas entre los grupos ya que las respuestas fueron bastante dispares. Cabe destacar el bajo grado de consenso en general al responder esta pregunta, lo cual indicaría una elevada falta de acuerdo social acerca de que es lo que realmente permitiría mejorar la capacidad tecnológica del país. Lo único que aparece como de común acuerdo es que el gobierno y las empresas son quienes debieran invertir más dinero en el desarrollo de tecnología para mejorar los productos y procesos de manufactura chilenos.

Finalmente se indagó acerca de las posibles causas del atraso tecnológico nacional, diagnóstico no fácil considerando que es un problema sistémico multicausal, con raíces técnicas, económicas y sociales. De las respuestas obtenidas destacan las preferencias por adjudicar la responsabilidad del bajo nivel de desarrollo de tecnología nacional al gobierno (68\%) y a las empresas (60.8\%), agentes que, de acuerdo con lo que se mostró previamente, destinarían pocos recursos monetarios al desarrollo de tecnología chilena, aparentemente por debajo de lo que sería socialmente conveniente. Bajo el rango de $50 \%$ de las preferencias los encuestados responsabilizaron a la mala educación escolar en Chile (47.5\%), a las universidades que también debieran contribuir con más recursos monetarios (35.4\%) y a la mala educación de los técnicos (25.4\%). El resto de las alternativas presentaron una proporción menor al $20 \%$ por lo cual no serían relevantes en el atraso tecnológico nacional.

Para determinar la percepción de los encuestados acerca de los motivos que existirían para explicar las posibles causas del atraso tecnológico nacional, se llevó a cabo el análisis factorial con extracción por componentes principales de los datos de esta batería, la cual tenía 10 respuestas posibles. Las pruebas KMO y de Barlet de este análisis demostraron ser estadísticamente significativas. A partir de este análisis se extrajeron cuatro componentes que agruparon las causas de este atraso y que colectivamente retienen un $62.8 \%$ de la varianza de los datos. El primer componente retuvo un $23.9 \%$ de la varianza total, se fundamenta en la percepción de los encuestados acerca de lo deficiente que es la educación en Chile en general (escolar, técnica y universitaria). El segundo corresponde a la baja inversión en tecnología que realizan las universidades y las empresas nacionales, el cual retuvo un $15.1 \%$ de la varianza de los datos. El tercer componente considera la baja inversión en tecnología por parte del Gobierno, combinado con la percepción de que necesitamos desarrollar más tecnología autóctona; este retuvo un $12.5 \%$ de la varianza. El cuarto componente está constituido por dos respuestas que tienen una connotación pesimista respecto a la capacidad del país por hacer más tecnología propia: el país es muy chico y no tenemos la capacidad para hacerlo y creo que no somos capaces de hacer más, lo cual es un tanto preocupante por ser estas expresiones reflejo de la cultura nacional acerca del desarrollo de 
tecnología. Este último factor rotado explica un $11.3 \%$ de la varianza de la data.

Al analizar estos datos por edad, sexo y nivel socioeconómico se obtuvieron resultados inconsistentes, con distintos grupos justificando este atraso por distintas causas, lo que podría estar indicando falta de consenso respecto a este fenómeno. No obstante, la causa que suscita mayor acuerdo es la baja inversión en tecnología que hace el gobierno, seguida por la baja inversión de las empresas en esta actividad. La primera de estas causas se puede considerar una característica de la sociedad chilena que tendería a exigir al gobierno un mayor involucramiento en casi todos los ámbitos, particularmente cuando no hay una respuesta alternativa fácil. Por otro lado, es correcto que a nivel nacional se deba aumentar la inversión en desarrollo tecnológico, ya que Chile invierte tan solo un $0.4 \%$ del PIB en Investigación y Desarrollo Tecnológico mientras que, en el otro extremo de la OCDE, Corea del Sur invierte 4\% de su PIB en esto (OCDE, 2013).

\section{DISCUSIÓN Y CONCLUSIONES}

De los resultados obtenidos en este estudio se pueden derivar las diversas percepciones que evidencian las personas sobre la tecnología en Chile.

En primer lugar, el estudio mostró diferencias estadísticamente significativas entre mujeres mayores de grupo socioeconómico bajo y hombres jóvenes de grupo socioeconómico alto en la forma en que conceptualizan la tecnología. Por lo tanto, una frase tal como el gobierno comenzará a impulsar el desarrollo de tecnología nacional tendrá una interpretación totalmente diferente por parte de estos dos individuos. Teniendo en cuenta las dispares interpretaciones dadas al concepto, cada vez que se considere el tema de la tecnología, ya sea para discusión pública o para efectos de comunicación, es altamente necesario definir claramente lo que se entendería por tecnología dentro del contexto del mensaje que se quiere formular.
Por otro lado, este estudio ha contribuido a clarificar que, a pesar de que se cree que las nuevas tecnologías y su innovación afectarán positivamente a las personas, esto no sería percibido de la misma forma por todos los sujetos, pues las mujeres mayores de clase socioeconómica baja tienden a otorgarle a la tecnología una importancia menor en su vida que los hombres jóvenes de clase socioeconómica alta. Por lo tanto, aquellas políticas públicas destinadas a la innovación y al desarrollo tecnológico nacional tenderán a ser percibidas como más beneficiosas y obtener más apoyo por parte de los hombres jóvenes de clase alta que por las mujeres mayores de clase baja.

Respecto a los beneficios percibidos que la tecnología tendría para la vida diaria de las personas, este estudio permitió determinar que para los grupos socioeconómicos alto y medioalto la tecnología generaría un importante conjunto de beneficios, los cuales no son 
reconocidos de igual manera por los individuos pertenecientes a los grupos socioeconómicos bajo y medio-bajo. La explicación para ello sería por motivos económicos, de educación y laborales. Junto con estos hallazgos, a lo largo de esta investigación se pudo constatar la existencia de un reducido grupo de encuestados que manifiesta una percepción negativa respecto a lo que la tecnología aporta a su vida, los beneficios que tiene la misma y que la pobre condición tecnológica actual del país responde a que no se puede hacer más al respecto, corroborando la alienación que produciría la tecnología en los individuos según Marx (ver Wendling 2009). Ante esto, se recomienda que las políticas gubernamentales de incentivo a la innovación y al desarrollo de tecnología autóctona se orienten hacia los grupos de menores recursos económicos, a fin de ayudarlos a beneficiarse a partir del desarrollo tecnológico.

Respecto a quién o quiénes debieran tener una mayor relevancia en el desarrollo de tecnología en Chile, este estudio muestra que después del Gobierno son las empresas, seguidas por las universidades, las instituciones a las cuales se les asigna una alta importancia en el desarrollo de tecnología nacional; instituciones que no habrían respondido a tales expectativas ya que, según los encuestados, su nivel de inversión en estas materias sería menor a lo que los individuos consideran como conveniente.

A partir de los resultados de la presente investigación es posible reconocer que no existe un consenso entre los individuos encuestados respecto a las causas específicas que darían lugar a nuestro relativamente bajo nivel de desarrollo tecnológico, con la excepción del rol gravitante que le cabe al gobierno. No obstante, las causas que los sujetos atribuyen a esto se pueden agrupar en cuatro tipos: mala educación en general, la baja inversión en tecnología por parte de universidades y empresas, baja inversión del gobierno en tecnología, y una visión fatalista que indica que en el país no se puede hacer más de lo que ya se hace. Diversos individuos y grupos reconocen el rol secundario de los laboratorios nacionales en el desarrollo tecnológico nacional, los cuales son muy pocos y se encuentran bajo la responsabilidad del gobierno para su funcionamiento $\mathrm{y}$ financiamiento (Weinstein, 2002).

Cabe destacar el amplio consenso existente a nivel colectivo de que la acción del gobierno y su baja inversión para el desarrollo de tecnología autóctona serían las principales causas del bajo nivel de desarrollo nacional en materias tecnológicas. Esto es coincidente con lo que sostiene Castells (1999; citado por Gainza, 2003) de que "el Estado ha sido crucial para fomentar o estancar el desarrollo y la introducción de tecnologías, así como también tiene un papel muy importante en el desarrollo de la capacidad innovadora de sus sociedades" (p. 133). De acuerdo con esto, la sociedad chilena consideraría positivo que el gobierno destinara mayores recursos a la innovación tecnológica, dirigidos a financiar las actividades de los centros y laboratorios nacionales, y la investigación tecnológica de las universidades. Esto debería ser considerado por las autoridades para destinar más recursos al desarrollo de tecnología, apoyando especialmente a las 
Maldifassi, J. (2018). Percepción social de la tecnología en Chile. Revista de Sociología 33(1), 39-57. doi: 10.535/0719-529X.2018.51107

universidades y los laboratorios nacionales. Asimismo, se deben revisar las políticas existentes para incentivar a que empresas y universidades destinen más recursos para este mismo fin, intentando hacer llegar a los grupos socioeconómicos menos privilegiados los beneficios de tal desarrollo tecnológico.

\section{REFERENCIAS}

Basiuk, V. (1977). Technology, world politics \& American policy. Nueva York, NY: Columbia University Press.

Berger, P. L., \& Luckmann, T. (1997). La construcción social de la realidad. Buenos Aires, Argentina: Amorrortu Editores.

Bijker, W. E. (2009). Social construction of technology. En J. K. B. Olsen, S. A. Pedersen, \& V. F. Hendricks (Eds.), A companion to the philosophy of technology (pp. 88-94). Massachusetts, MA: Blackwell Publishing, Malden.

Bloor, D. (1999). Anti-Latour. Studies in history and philosophy of science, 30(1), 81-112. doi: 10.1016/S0039-3681(98)00038-7

Castells, M. (1999). La era de la información: Economía, sociedad y cultura. Volumen III fin de milenio. Madrid, España: Alianza Editorial.

Consejo Nacional de Ciencia y Tecnología de México (1998). Encuesta sobre la percepción pública de la ciencia y la tecnología en México. Recuperado de http://www.siicyt.gob. $m x /$ siicyt/docs/Estadis ticas2/encuesta_percepcion_1998.html

Davis, F. D. (1989). Perceived usefulness, perceived ease of use, and user acceptance of technology, MIS Quarterly, 13(3), 319-340. doi: $10.2307 / 249008$

Dosi, G., Teece D. J., \& Chytry, J. (1998). Technology, organization and competitiveness: Perspectives on industrial and corporate change. Oxford, Inglaterra: Oxford University Press.
Flores, L. (1992). La tecnología en el contexto de la cultura latinoamericana. En E. Sabrosky (Comp.), Tecnología y modernidad en Latinoamérica: Etica, política y cultura (pp. 19-23). Santiago de Chile: Ediciones Pedagógicas Chilenas.

Friedman, A. (2011). Perception: A cognitive sociological approach. En F. Clement, \& L. Kaufman (Eds.), La sociologie cognitive. Paris, Francia: Maison des Sciences de l'Homme.

Gainza, C. (2003). Sociedad, estado y tecnología: ¿Qué pasa hoy con nuestras sociedades?. Revista de Sociología, 17(1), 124-146. doi: 10.5354/0719-529X.2003.27794

Gill, I. S., Guasch, J. L., Maloney, W. F., Perry G., \& Schady, N. (2005). Cerrar la brecha en educación y tecnología. Bogotá, Colombia: Banco Mundial

Gordon, J. R. (1987). A diagnostic approach to organizational behavior (2da ed.). Boston, MA: Allin and Bacon Inc.

Klein, H. K., \& Kleinman, D. L. (2002). The social construction of technology: Structural considerations. Science technology and human values, 27(1), 28-52. doi: 10.1177/016224390202700102

Kotler, P., Armstrong, G., Cámara Ibáñez D., \& Cruz Roche, I. (2004). Marketing. (10ma ed.). Madrid, España: Pearson Prentice Hall. Law, J. (2002). Aircraft stories: Decentering the object in technoscience. Londres, Inglaterra: Duke University Press. 
Maldifassi, J. (2018). Percepción social de la tecnología en Chile. Revista de Sociología 33(1), 39-57. doi: 10.535/0719-529X.2018.51107

Macionis, J. J. (1989). Sociology (2da ed.). New Jersey, EEUU: Prentice Hall.

Maldifassi, J., \& Canessa, E. (2009). Information technology in Chile: How perceptions and use are related to age, gender, and social class. Technology in Society, 31(3), 273-286. doi: 10.1016/j.techsoc.2009.03.006

Malhotra, N. K. (2004). Investigación de mercados: Un enfoque aplicado (4ta ed.). Naucalpan de Juárez, México: Pearson Prentice Hall.

Mannheim, K. (1941). Ideología y utopía: Introducción a la sociología del conocimiento. Ciudad de México, México: Fondo de Cultura Económica.

Merton, R. K. (1973). The sociology of science: Theoretical and empirical investigations. Chicago, IL: The University of Chicago Press.

Nelson, R. N. (1993). National innovation systems: A comparative analysis. Nueva York, NY: Oxford University Press.

Northcraft, G. B., \& Neale, M. A. (1990). Organizational behavior: A management challenge. Chicago, IL: The Dryden Press.

Organization for Economic Cooperation and Development (2013). OECD science, technology and industry scoreboard: Innovation for growth. Paris, Francia: OECD Publishing.

Pinch, T., \& Bijker, W. (1987). The social construction of facts and artifacts: Or how the sociology of science and the sociology of technology might benefit each other. En W. Bijker, T. Hughes, \& T. Pinch (Eds.), The social construction of technology systems: New Directions in the sociology and history of technology (pp. 17-50). Cambridge, MA: MIT Press

Pursell, C. W. Jr. (1990). Technology in America: $A$ history of individuals and ideas (2da ed.). Preston, ID: MIT Press.

Rogers, E. M. (1995). Diffusion of innovations (4ta ed.). Nueva York, NY: The Free Press.

Roth, R. (2010). Marx on technical change in the critical edition. The European Journal of the History of Economic Thought, 175), 12231251. doi: 10.1080/09672567.2010.522239

Silva-Peña, I., Borrero, A. M., Marchant, P., González, G., \& Novoa, D. (2006). Percepciones de jóvenes acerca del uso de las tecnologías de información en el ámbito escolar. Última Década, 14(24), 37-60.

Thomas, W. I., \& Thomas D. S. (1928). The child in America: Behavior problems and programs. Nueva York, NY: Knopf Publishers.

Weinstein, A. (2002). Análisis comparativo del Sistema Nacional de Innovación con el propósito de mejorar su efectividad. (Tesis de pregrado no publicada). Pontificia Universidad Católica de Chile, Santiago, Chile.

Wendling, A. E. (2009). Karl Marx on technology and alienation. Londres, Inglaterra: Palgrave Macmillan.

Manuscrito recibido: 10-01-2018 Manuscrito aceptado: 15-04-2018 